\title{
CARACTERIZACIÓN DEL FLUJo DE AIRE EN COLINAS PARA EL EMPLAZAMIENTO DE PARQUES EÓLICOS
}

\section{Characterization OF AIR FLOW IN HILlS FOR EMPLACEMENT OF WIND FARMS}

\author{
Fran Reinoso-Avecillas ${ }^{1, *}$, Nelson Jara-Cobos ${ }^{1}$, Pablo Gómez-del Pino ${ }^{2}$ y \\ César Nieto-Londoño ${ }^{3}$
}

\section{Resumen}

Este artículo presenta el análisis del comportamiento de flujo de aire sobre una colina en la zona inferior de la capa límite atmosférica, empleando un software de propósito general de Dinámica de Fluidos Computacional (CFD). Mediante un análisis comparativo entre los resultados obtenidos en las simulaciones y los datos experimentales disponibles de colinas estandarizadas con diferentes topografías; se ha logrado validar convenientemente el procedimiento de simulación. A continuación; se simuló bidimensionalmente el flujo de aire sobre una loma real; se compararon los resultados numéricos con estudios científicos similares y de manera puntual con los datos de operación del parque eólico instalado en el sitio, logrando caracterizar con cierto grado de detalle el campo de velocidades y de energía cinética turbulenta a lo largo del dominio; además de obtener coincidencias cercanas en cuanto su magnitud con variaciones en el orden de 1 a $2 \mathrm{~m} / \mathrm{s}$ para la velocidad horizontal media.

Palabras clave: energía eólica, aerogenerador, dinámica de fluidos computacional, flujo de aire, simulación.

\section{Abstract}

This article presents the analysis of the behavior of air flow on a hill in the bottom of the atmospheric boundary layer, using a general purpose software Computational Fluid Dynamics (CFD). Through a comparative analysis of the results of the simulations and the experimental data with different topographies standardized hills available; It has been achieved conveniently validate the simulation procedure. Next; is two-dimensionally simulated air flow over an actual ridge; the numerical results with similar scientific studies and in a timely manner with the operating data of wind farm installed on site, achieving characterize with some detail the velocity field and turbulent domain along the kinetic energy is compared; plus get close coincidences as its magnitude with variations in the order of 1 to $2 \mathrm{~m} / \mathrm{s}$ for the average horizontal velocity.

Keywords: Wind Power, Wind Turbine, Computational Fluid Dynamics, wind flow, Complex Terrain.

\footnotetext{
${ }^{1, *}$ Grupo de Investigación y Desarrollo en Simulación y Toma de Decisiones GID - STD. Universidad Politécnica Salesiana - Ecuador. Autor para correspondencia freinoso@ups.edu.ec

${ }^{2}$ Departamento de Mecánica. Universidad Nacional de Educación a Distancia - España.

${ }^{3}$ Facultad de Ingeniería Mecánica. Universidad Pontificia Bolivariana, Medellín - Colombia.
}

Recibido: 08-12-2015, aprobado tras revisión: 23-05-2016.

Forma sugerida de citación: Reinoso, F.; Jara, N.; Gómez, P.; Nieto, C. (2016). «Caracterización del flujo de aire en colinas para el emplazamiento de parques eólicos». InGENIUS. N. ${ }^{\circ} 15$, (Enero-Junio). pp. 17-26. ISSN: 1390-650X. 


\section{Introducción}

El flujo del viento se ve afectado por factores como la rugosidad y la topografía del terreno, que tienden a producir perturbaciones en su movimiento. La presencia de lomas, por ejemplo, puede ser por un lado beneficiosa ya que la velocidad del viento aumenta al llegar a la cima, pero por otro lado, pendiente abajo de la colina, la turbulencia se ve incrementada; y se pueden producir efectos de recirculación. Este comportamiento no permite conocer con detalle el desarrollo de los perfiles de velocidad y la energía cinética turbulenta del viento; por lo que existe en la actualidad un gran interés sobre el estudio del flujo de aire en lomas o terrenos complejos.

\subsection{Flujo de aire en terrenos homogéneos}

La velocidad del viento en terrenos homogéneos; depende principalmente de la resistencia que presente la superficie del terreno a su paso y cuyo perfil logarítmico de velocidad está totalmente definido para la capa de la troposfera; está representado como $u(z)$ [1], [2], [3], [4] y responde a la Ecuación 1.

$$
u(z)=\frac{u^{*}}{k}\left[\ln \frac{z}{z_{0}}-\Psi\left(\frac{z}{L}\right)+\Psi\left(\frac{z_{0}}{L}\right)\right]
$$

Donde,

$u(z)=$ velocidad del viento a la altura $z$ por encima de la superficie del suelo.

$u^{*}=$ velocidad de fricción.

$\tau=$ esfuerzo superficial y $\rho$ la densidad del aire; donde $\tau=\rho u^{2}$.

$k=$ constante de Von Karman, que generalmente toma el valor de $0.40[5]$.

$L=$ longitud de Monin-Obukhov.

$\Psi=$ función universal adimensional del perfil, que depende de la estabilidad atmosférica.

\subsection{Flujo de aire en terrenos no homogéneos}

Un terreno no homogéneo, se caracteriza por tener una amplia zona horizontal no uniforme, que en general dan lugar a la formación de colinas y valles. En este caso, el comportamiento del flujo de aire en la capa límite atmosférica se ve influenciada por los diferentes accidentes que posee el terreno; generando variaciones en su dimensión, gradientes de temperatura, y la adhesión de nuevos flujos considerados como turbulentos. En consecuencia; la presencia de distintos tipos de topografía de terreno afecta de manera particular al desarrollo de los perfiles de velocidades y de energía cinética turbulenta del flujo de aire [4], [6], [7].

Para su estudio, los autores Gripphins y Middelton presentan una estandarización de lomas en dos dimensiones (Fig. 1), que responden a la función paramétrica de comportamiento senoidal de acuerdo con la Ecuación 2.

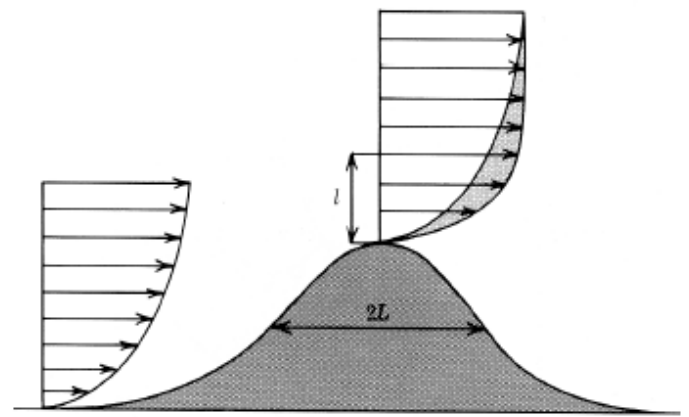

Figura 1. Perfil vertical de velocidades [4].

$$
z_{s}=\left\{\begin{array}{l}
\frac{H}{2}\left[1+\cos \frac{\pi x}{2 L}\right], \quad-2 L<x<2 L \\
0, \quad|x| \geq 2 L
\end{array}\right.
$$

El parámetro de estandarización es la relación $H / L$, donde $H$ es la altura de la loma, $L$ es la longitud de la loma; de esta manera, se definen cuatro lomas estándar con los siguientes valores de $H / L: 0.75 ; 1 ; 2 ;$ y 4 , la altura típica referencial del modelo es de $H=60 \mathrm{~m}$ [8].

En los valles, el aire caliente se eleva y genera una zona de baja presión, haciendo que el aire más fresco ascienda ladera arriba, es lo que se conoce como viento de valle. La estandarización de los valles sigue criterios similares a las lomas en función de los parámetros $H$ y $L$. Según los estudios realizados por Maurizi, se definen como valles poco profundos cuando la relación media de $H / L=0.25$ y valles profundos en los cuales se presenta una relación media semejante a $H / L=0.66$ [9].

\subsection{Ecuaciones de gobierno del flujo}

Para el estudio de flujo de aire sobre colinas se han desarrollado una variedad de modelos y técnicas numéricas, que interpretan y resuelven las ecuaciones fundamentales del flujo de fluidos como son la conservación de la masa, la conservación de la energía y de la cantidad de movimiento, para flujo viscoso representadas en las ecuaciones de Navier-Stokes, Ecuación 3, 4 [10], [11].

$$
\begin{gathered}
\frac{D \rho}{D t}+\rho(\nabla \cdot V)=0 \\
\rho \frac{D V}{D t}=-\nabla p+\nabla \cdot \overline{\bar{\tau}}^{\prime}+\rho f
\end{gathered}
$$

\subsection{Turbulencia}

La turbulencia puede ser caracterizada como el movimiento caótico del fluido; su incremento está en directa dependencia de la relación entre las fuerzas de inercia y las fuerzas viscosas en el fluido [12]. La energía cinética $k$ de los remolinos de mayor escala fluyen 
hacia los de menor escala a través de un mecanismo de cascada y está dada por el coeficiente de disipación $\epsilon$ de la energía cinética $k[13]$. La intensidad de turbulencia $(I)$, se define como la relación entre desviación estándar y la media de los datos correspondientes a las series de velocidad de viento en un período de tiempo corto (no mayor a una hora) y por convención usualmente igual a diez minutos [14]. Considerando una desviación estándar $\sigma_{u}=2.5 u^{*}$ cerca del suelo se obtiene el siguiente modelo de la turbulencia, como se muestra en la Ecuación 5.

$$
I_{u}=\frac{\sigma_{u}}{\bar{u}}
$$

Donde,

$\bar{u}=$ velocidad media del viento.

$\sigma_{u}=$ desviación estándar.

\subsection{Modelo de turbulencia $k-\epsilon$ estándar}

El modelo de turbulencia $k-\epsilon$ propuesto originalmente por Launder y Spalding y ampliamente utilizado debido a su robustez y economía en la resolución de flujos relativamente simples; incorpora dos ecuaciones de balance en derivadas parciales en las que interrelacionan la energía cinética del fluido turbulento $k$ y su velocidad de disipación $\epsilon$ [2], [3], [15], [16].

$$
\begin{aligned}
\rho \frac{\partial k}{\partial t}+\rho \bar{u}_{i} \frac{\partial k}{\partial x_{j}} & =\tau_{\ddot{v}} \frac{\partial \bar{u}_{i}}{\partial x_{j}}-\rho \epsilon \\
& +\frac{\partial}{\partial x_{i}}\left[\left(\mu+\frac{\mu_{t}}{\sigma_{k}}\right) \frac{\partial k}{\partial x_{i}}\right] \\
\rho \frac{\partial \epsilon}{\partial t}+\rho \bar{u}_{i} \frac{\partial \epsilon}{\partial x_{i}} & =C_{\epsilon 1} \frac{\epsilon}{k} \tau_{\ddot{v}} \frac{\partial \bar{u}_{i}}{\partial x_{j}}-C_{\epsilon 2} \rho \frac{\epsilon^{2}}{k} \\
& +\frac{\partial}{\partial x_{i}}\left[\left(\mu+\frac{\mu_{t}}{\sigma_{\epsilon}}\right) \frac{\partial \epsilon}{\partial x_{i}}\right]
\end{aligned}
$$

Donde la Ecuación 6 representa el transporte de la energía cinética $k$ y la Ecuación 7 , es la ecuación de la velocidad de disipación $\epsilon$, respectivamente.

$C_{\mu}, C_{1 \epsilon}, C_{2 \epsilon}, \sigma_{k}, \sigma_{\epsilon}$; son el conjunto de constantes de identificación, que para el modelo estándar se han fijado los siguientes valores por defecto: $C_{\mu}=0.09$; $C_{1 \epsilon}=1.44 ; C_{2 \epsilon}=1.92 ; \sigma_{k}=1 ; \sigma_{\epsilon}=1.3$.

\section{Materiales y métodos}

\subsection{Características del problema a simular para la validación}

Se realizó la validación del procedimiento mediante la simulación del flujo de aire sobre la colina de Askervein, usando para ello el paquete computacional Ansys Fluent. Mediante simulaciones bidimensionales 2D aplicadas a las secciones A-A y AA-AA, con una longitud transversal aproximada de $2 \mathrm{~km}$ como se muestra en la Figura 2, se compararon satisfactoriamente los datos experimentales de la colina de Askervein con los resultados numéricos de la simulación [17].

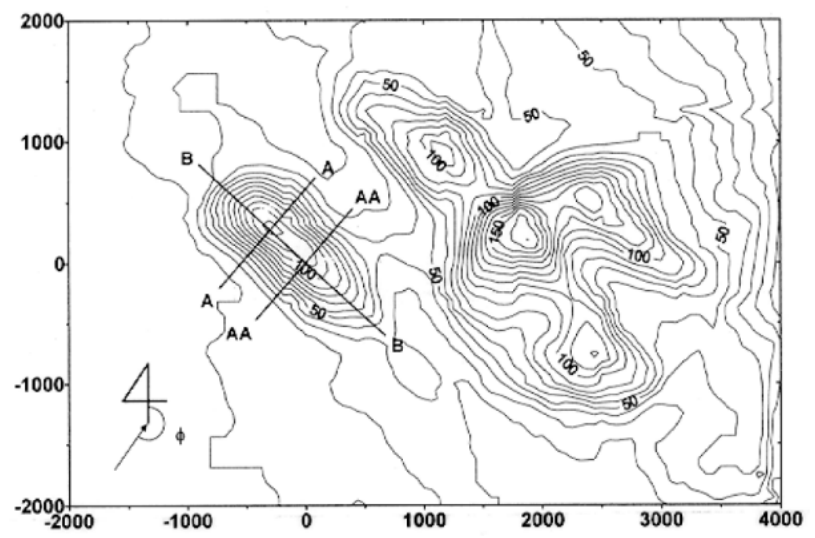

Figura 2. Topografía colina de Askervein - líneas referenciales [17].

\subsection{Características de la zona de estudio}

La zona de interés está situada al sur de Ecuador, con coordenadas UTM: N9558404, E693030 y N9556225, E693635; en donde se encuentra instalado el Parque Eólico Villonaco a $2720 \mathrm{~m}$ sobre el nivel del mar; cuenta con 11 aerogeneradores tripala de eje horizontal de 1.5 MW de potencia; con altura de buje de $65 \mathrm{~m}$, y una capacidad de generación total de 16.5 Mw [18], [19].

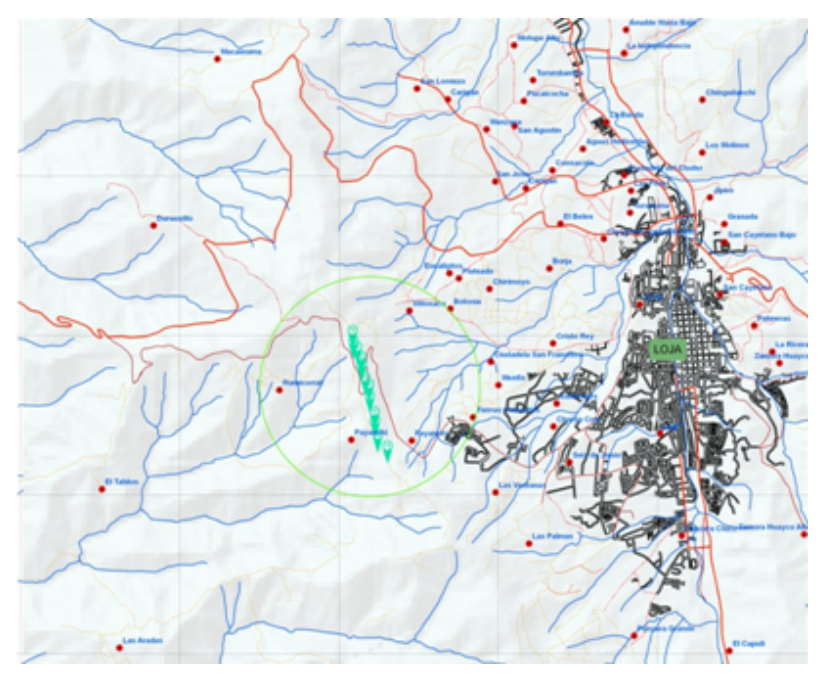

Figura 3. Ubicación de la zona de estudio [18].

\subsection{Modelado del problema}

\subsubsection{Software utilizado}

En la generación de la superficie del modelo, se utilizó el software Autodesk Inventor Series V 14, y la simulación del problema mediante el paquete Fluent de ANSYS, para resolver las ecuaciones de Navier Stokes sobre la malla. 


\subsubsection{Software utilizado}

El modelo físico de la colina de Askervin, tiene una longitud $L=6000 \mathrm{~m}$ y una altura máxima $H=459 \mathrm{~m}$. Se genera el dominio computacional que contenga a la loma, con una altura $6 \mathrm{H}$, y una longitud de $10 \mathrm{H}$ a partir del perfil obtenido; como se observa en la Figura 4. Un procedimiento similar se sigue para la generación del modelo físico de la colina del sitio de interés.

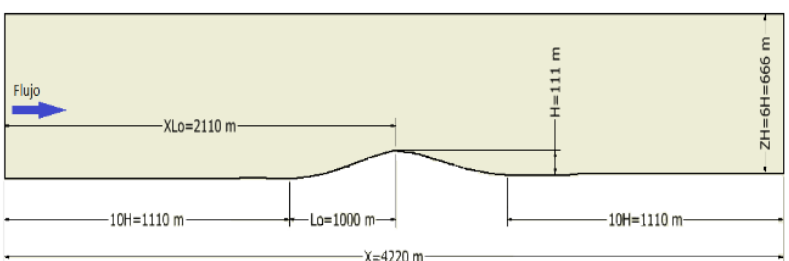

Figura 4. Dimensiones del dominio computacional de la colina estudiada.

\subsubsection{Generación de la malla}

Se utiliza una malla no estructurada de volúmenes finitos en forma de tetraedros a partir de $15 \mathrm{~m}$ por encima del suelo y hasta $600 \mathrm{~m}$ (parte superior de frontera), donde suponemos que la superficie tiene poco efecto sobre el flujo y la condición de estabilidad es neutral. Desde la superficie (suelo) a la parte inferior de la rejilla no estructurada, se utilizó una rejilla prismática estructurada para refinar la región dentro de los primeros $15 \mathrm{~m}$ sobre el suelo, y las mallas no estructuradas se vieron obligadas a coincidir en $15 \mathrm{~m}$ de altura (Fig. 5). Dentro de esta región de $15 \mathrm{~m}$, se definen cinco estratos estructurados con diferentes alturas de las rejillas $\Delta Z S$ que se fusionaron con la red no estructurada anterior.

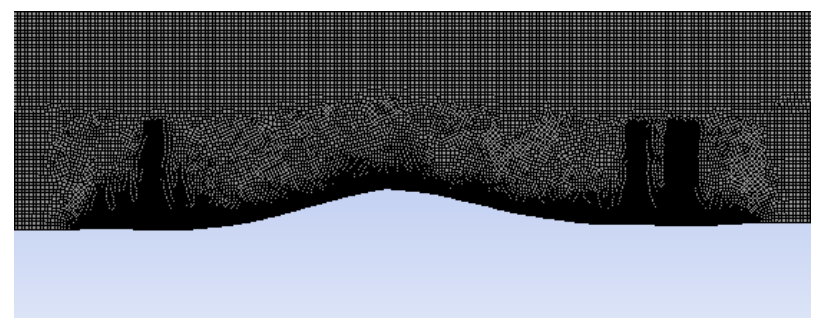

Figura 5. Mallado 2D de dominio computacional de colina Askervin.

Para el sitio de interés (Fig. 6); se ha generado una malla en dos dimensiones 2D, con 106086 nodos y 105079 elementos. Los elementos de la malla son tetraedros para toda la superficie con tamaño fino.

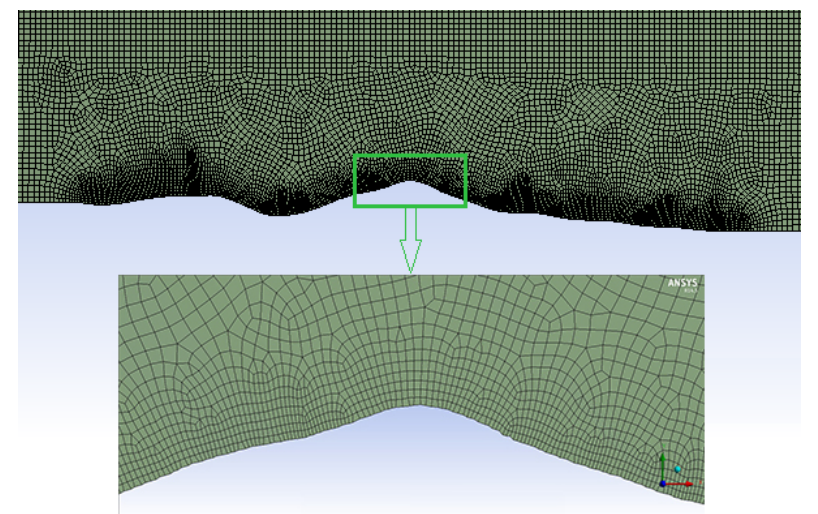

Figura 6. Mallado 2D del dominio computacional del sitio de interés.

\subsubsection{Condiciones de contorno}

Las propiedades de cada frontera del dominio computacional determinadas, tanto para el modelo Askervin como para el sitio de interés, se presentan en la Tabla 1 . El valor de altura de rugosidad de la superficie $z_{0}=0.03 \mathrm{~m}$.

Tabla 1. Condiciones de contorno para línea A-AAskervin.

\begin{tabular}{lcl}
\hline $\begin{array}{l}\text { Nombre de } \\
\text { Arista }\end{array}$ & Variable & $\begin{array}{l}\text { Datos } \\
\text { característicos }\end{array}$ \\
\hline \multirow{2}{*}{ Inlet } & Velocity-inlet & $\begin{array}{l}\text { Velocidad: } 9 \mathrm{~m} / \mathrm{s} \\
\text { Intensidad de turbulencia: } 9 \% \\
\text { Longitud de escala } 1 \mathrm{~m} .\end{array}$ \\
\hline \multirow{2}{*}{ Outlet } & Pressure-Outlet & $\begin{array}{l}\text { Intensidad de turbulencia: } 9 \% \\
\text { Longitud de escala: } 1 \mathrm{~m} .\end{array}$ \\
\hline Perfil & Wall & Rugosidad del terreno: $0.02 \mathrm{~m}$. \\
Cielo & Wall & Tensión: constante \\
\hline
\end{tabular}

\subsubsection{Modelado de turbulencia}

El modelo de turbulencia utilizado para este trabajo es $k-\epsilon$; conveniente para flujos geofísicos turbulentos con número de Reynolds alto [15]. Las constantes usadas corresponden al modelo estándar: $C_{\mu}=0.09 ; \sigma_{k}=1$; $\sigma_{\epsilon}=1.3 ; \sigma_{\epsilon 1}=1.44$ y $\sigma_{\epsilon 2}=1.92$.

\section{Resultados}

\subsection{Validación del procedimiento}

Se realizó mediante la simulación numérica de dos casos de colinas con datos conocidos. Para el primer caso de la colina de Askervin; con lo cual se pretende validar el procedimiento de simulación en todo el dominio; y para el segundo caso de estudio de una colina alargada de dimensiones normalizadas planteada por Rokones y Krostad; para verificar el comportamiento del desarrollo de los perfiles de velocidad horizontal y 
energía cinética turbulenta a lo largo de la colina en función de la variación de la pendiente de la misma.

\subsection{Simulación numérica del flujo de aire en la colina de Askervin. Sección A-A}

Una vez que la solución ha convergido, en la Figura 7 se presentan los resultados con los contornos de la componente horizontal de la velocidad en todo el dominio, en donde se puede apreciar el valor mínimo $0 \mathrm{~m} / \mathrm{s}$ en todo el perfil de la colina y el máximo $14.65 \mathrm{~m} / \mathrm{s}$ en la cima de la colina. En la Figura 8 se muestran los contornos de energía cinética turbulenta, con un valor mínimo de $0.002 \mathrm{~J} / \mathrm{kg}$ y un valor máximo de $0.395 \mathrm{~J} / \mathrm{kg}$.

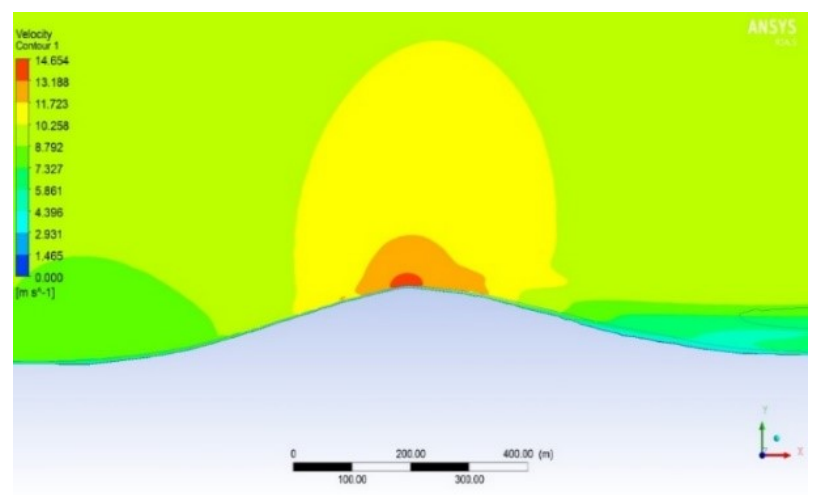

Figura 7. Contornos de velocidad en el dominio - colina de Askervin sección A-A.

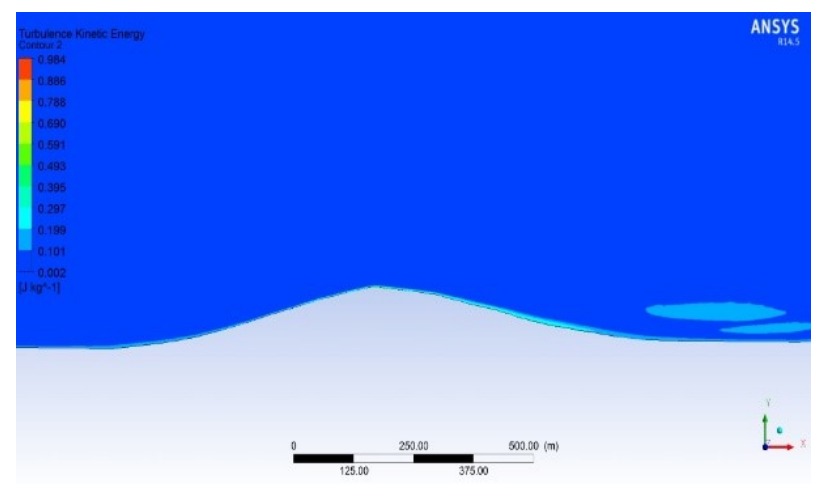

Figura 8. Contornos de energía cinética turbulenta en el dominio - colina de Askervin sección A-A.

En la Figura 9, se muestran los resultados presentados por los autores Forthofer y Butler [17] a una altura de $10 \mathrm{~m}$ del suelo, para la colina de Askervin. Se trata de una comparación entre valores obtenidos experimentalmente y resultados numéricos correspondientes a un método basado en la conservación de la masa y un método de tipo CFD.

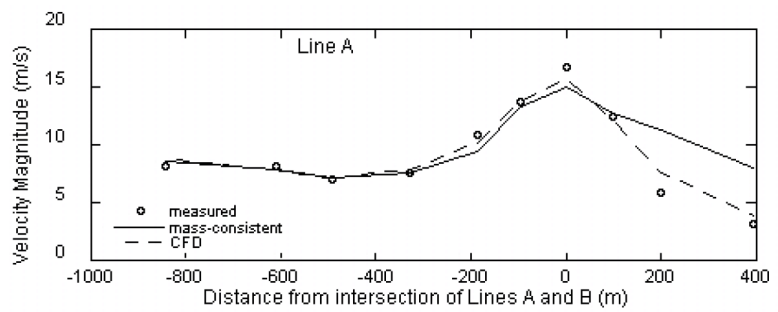

Figura 9. Velocidades de viento de colina Askervin sección A-A [17].

Los resultados numéricos correspondientes al método CFD de la Figura 9; son comparados con los obtenidos en este trabajo, observando un grado de acuerdo muy bueno, con errores en el orden de $1 \mathrm{~m} / \mathrm{s}$; como se muestra en la Figura 10.

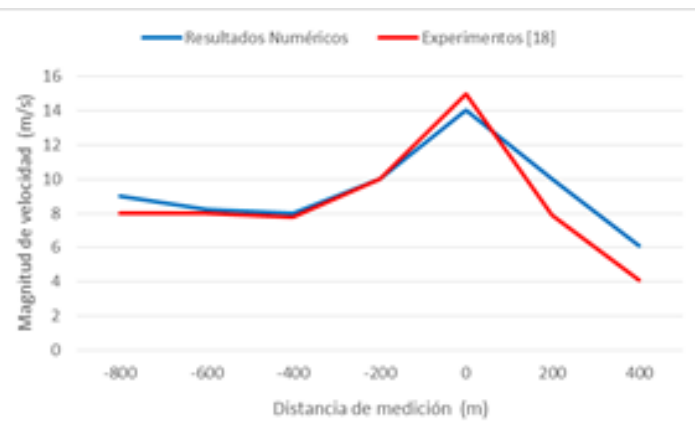

Figura 10. Comparación entre resultados numéricos de velocidad vs. datos experimentales para Askervin- sección A-A.

Siguiendo el mismo procedimiento establecido en el análisis de la velocidad del flujo de aire sobre la colina de Askervin; en la Figura 11 se presenta el comportamiento de la energía cinética turbulenta.

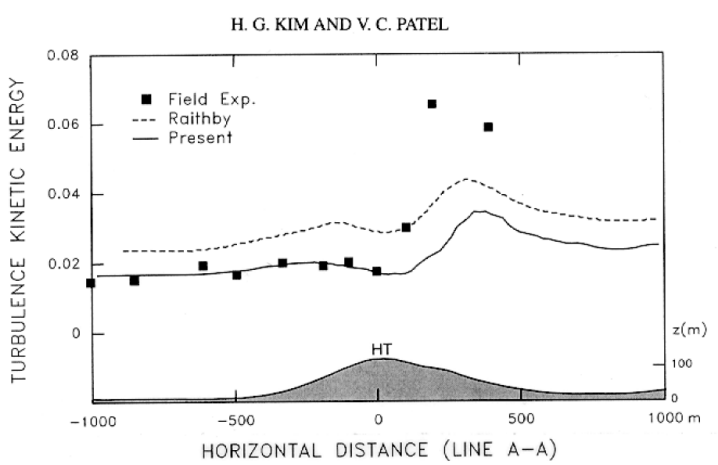

Figura 11. Perfiles de energía cinética turbulenta - colina de Askervin sección A-A [17].

En la Figura 12, se muestra una comparación entre los resultados numéricos correspondientes a la energía cinética turbulenta obtenidos en este trabajo y los valores experimentales presentados por Forthofer y Butler. En la cual se aprecia un grado de acuerdo relativamente bueno. Las principales diferencias se producen en la zona de pendiente debajo de la colina. 


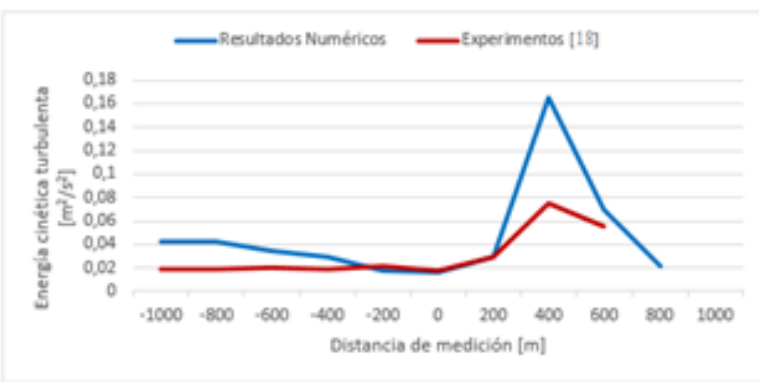

Figura 12. Comparación entre resultados numéricos de velocidad vs. datos experimentales para Askervin sección A-A.

Para completar el proceso de validación del procedimiento, se procede a la simulación numérica del flujo en una colina alargada (caso I) del modelo propuesto por Rokones y Krogstad [20]; que tiene por objeto realizar una validación más completa, tanto en la cima como en el valle de la colina, cuyas características se definen en la Tabla y cuyo perfil se muestra en la Figura 13.

Tabla 2. Condiciones de contorno para línea A-AAskervin.

\begin{tabular}{cccccc}
\hline Caso I & $\mathrm{H}[\mathrm{mm}]$ & $\mathrm{L}[\mathrm{mm}]$ & $\alpha_{\text {med }}\left[{ }^{\circ}\right]$ & $\alpha_{\text {máx }}\left[^{\circ}\right]$ & $\mathrm{Lp}[\mathrm{mm}]$ \\
\hline & 350 & 661 & 27.9 & 37.3 & 1424 \\
\hline
\end{tabular}

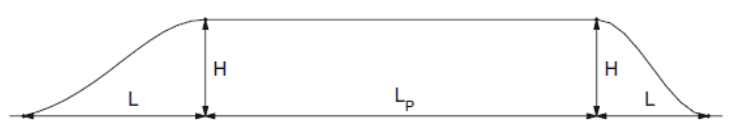

Figura 13. Dimensiones de la colina propuesta por Rokenes y Krogstad [20].

Se procede a determinar el dominio computacional de la colina del Caso I, con las dimensiones que se muestran en la Figura 14.

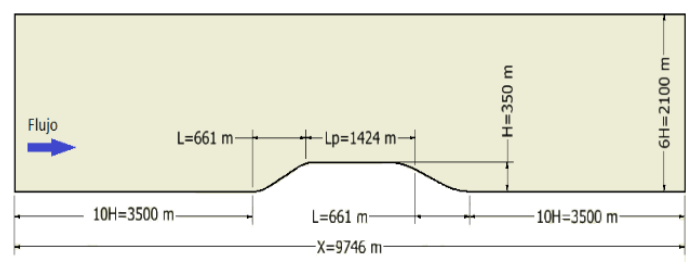

Figura 14. Dimensiones de la colina propuesta por Rokenes y Krogstad [20].

Se generó una malla 2D con las condiciones de entrada del flujo de $10.7 \mathrm{~m} / \mathrm{s}$ a una altura de $300 \mathrm{~m}$ y la rugosidad de terreno de $0.2 \mathrm{~m}$. La turbulencia tiene una intensidad de $9 \%$ a una longitud de escala $1 \mathrm{~m}$.

El modelo de turbulencia utilizado es $k-\epsilon$ estándar. Los contornos de la componente horizontal de la velocidad y de la energía cinética turbulenta resultantes del proceso de simulación se pueden observar en las Figuras 15 y 16. Las velocidades máximas son de $15.8 \mathrm{~m} / \mathrm{s}$ y la energía cinética turbulenta está en el orden de $2.40 \mathrm{~J} / \mathrm{kg}$.

Del análisis del desarrollo de los perfiles de velocidad y energía cinética turbulenta en cuatro posiciones estratégicas a lo largo del perfil de la colina y coincidentes con las posiciones establecidas en el Caso I: $\left(X / H_{1}=0.04 ; 0.9 ; 1.91 ;\right.$ y 4.91$)$; se observa que la velocidad media, presenta un comportamiento creciente a medida que se avanza pendiente arriba de la colina hasta llegar a su máximo en la cima. En la meseta de la loma, la velocidad permanece constante.

Como se observa en las Figuras 17, 18 y 19; para la posición $X / H_{1}=1.91$ que corresponde a la cima de la colina, se tienen variaciones máximas de $U / U_{r} \approx 0.22$, que corresponde a la velocidad media absoluta de $2.35 \mathrm{~m} / \mathrm{s}$. En la posición $X / H_{1}=4.91$ en la meseta de la colina, se tienen variaciones máximas de $U / U_{r} \approx 0.20$, que corresponde a la velocidad media absoluta de $2.14 \mathrm{~m} / \mathrm{s}$. Con esta comparación muestra un grado de acuerdo razonablemente bueno, entre los valores experimentales y los resultados numéricos.

En cuanto a la energía cinética turbulenta; se obtienen comportamientos similares a lo largo del perfil de la colina hasta prácticamente el final de la meseta; en cuanto al desarrollo de los perfiles, para todos los puntos de medición se observan un incremento en la turbulencia a medida que se acerca al suelo, lo que confirma en su totalidad los criterios planteados por Rokones y Krogstad, en su estudio del flujo de aire sobre colinas.

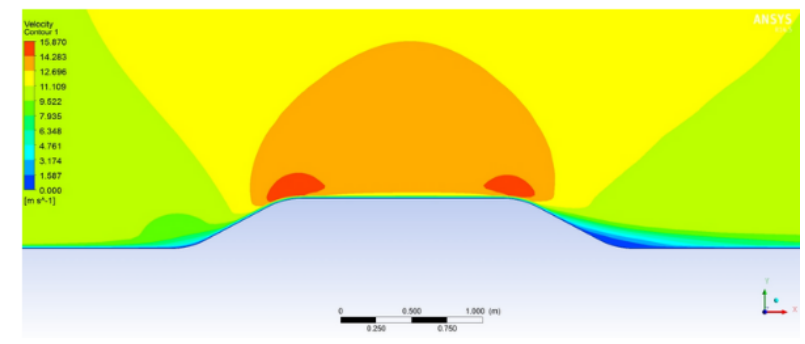

Figura 15. Contornos de velocidad en el dominio colina Caso I.

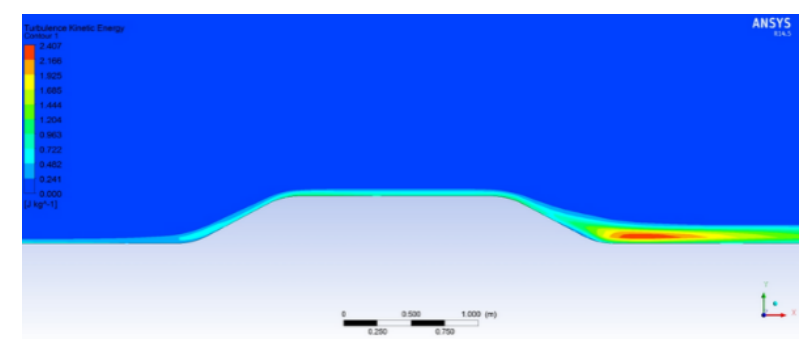

Figura 16. Contorno de energía cinética turbulenta en el dominio colina Caso I. 


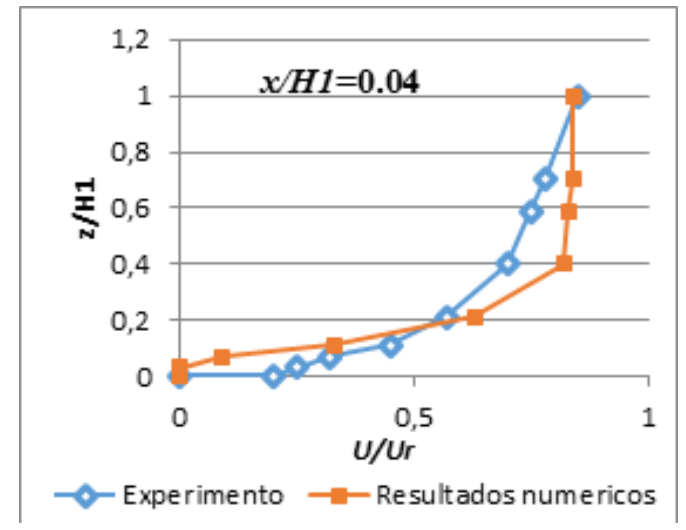

Figura 17. Comparación de resultados numéricos de velocidad vs. experimento de caso I.

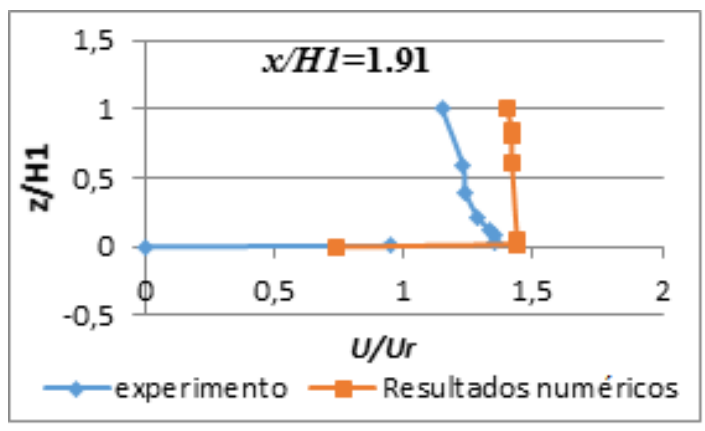

Figura 18. Comparación de resultados numéricos de velocidad vs. experimento de caso II.

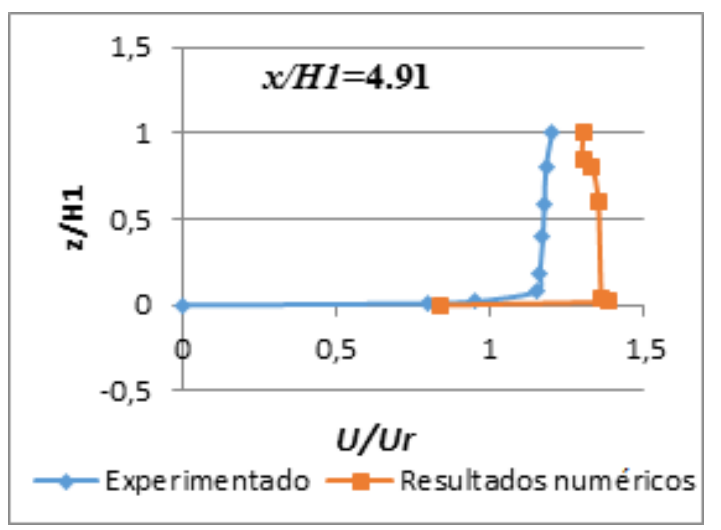

Figura 19. Comparación de resultados numéricos de velocidad vs. experimento de caso III.

\subsection{Simulación del sitio de interés}

Se realizó el estudio del flujo de aire sobre la colina seleccionada, mediante varias simulaciones bidimensionales del sitio de interés; para obtener información sobre el comportamiento de la velocidad y la intensidad de turbulencia a lo largo de la colina y comparar dichos resultados numéricos con estudios realizados sobre este tema.
En la Figura 20, se ha establecido las dimensiones de la colina del sitio de interés; con una de sus principales características de $H / L=0.75$ y pendiente de $23.5^{\circ}$. En la Figura 21, se muestra los puntos de análisis a lo largo del perfil de la loma; para los perfiles de velocidad y energía cinética turbulenta.

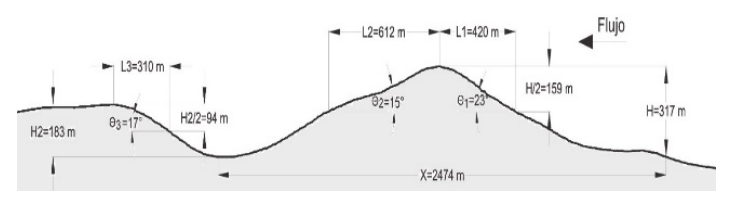

Figura 20. Dimensiones generales.

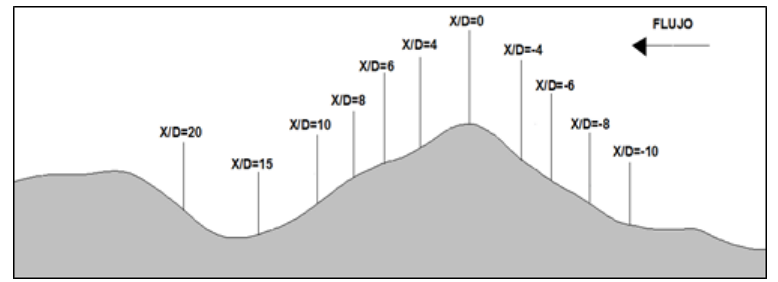

Figura 21. Ubicación de puntos de análisis a lo largo del perfil de la loma.

En la Figura 22, se presentan los resultados de contornos de la componente horizontal de la velocidad en todo el dominio, en donde se puede apreciar el valor máximo alrededor de $9.81 \mathrm{~m} / \mathrm{s}$ correspondientes a la cima de la colina.

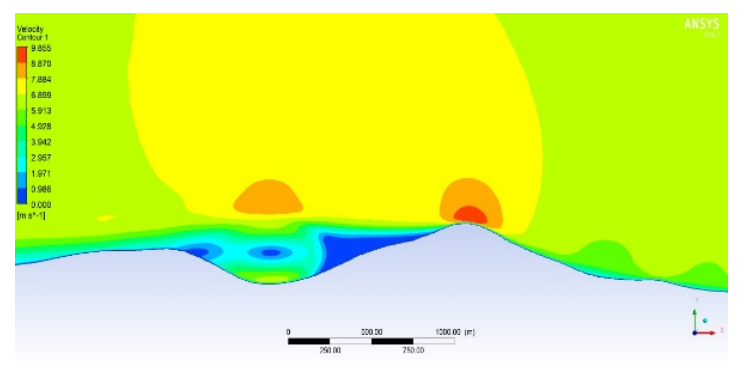

Figura 22. Contornos de velocidad en el dominio de sitio de interés.

El perfil de velocidades en $X / D=0$, correspondiente a la cima de la colina; obtenido mediante la simulación, se muestra en la Figura 23. En la cual se ha identificado, además, los valores puntuales de velocidad medidos en el sitio de interés.

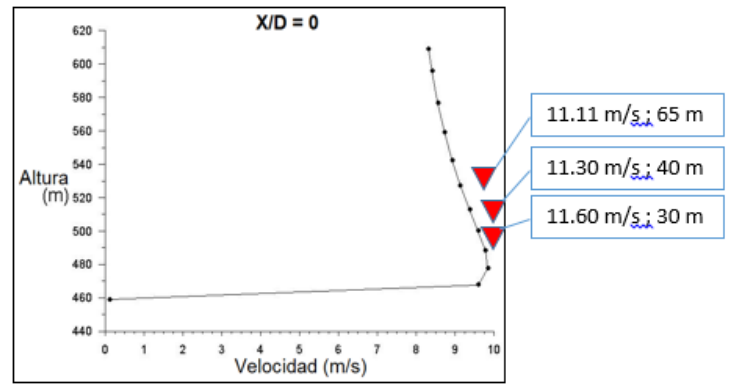

Figura 23. Perfil de velocidad en la cima de la colina $X / D=0$ en sitio de interés. 
Los perfiles de velocidades, pendiente arriba de la loma, representados en la Figura 24; son similares en cuanto a su forma; se puede observar que la velocidad tiene valores iniciales de $5.0 \mathrm{~m} / \mathrm{s}$. En la posición de máxima pendiente, es decir, a mitad de la colina el flujo todavía mantiene un retraso de $u=7.2 \mathrm{~m} / \mathrm{s}$ para $X / D=-4$; llegando a valores máximos de $9.8 \mathrm{~m} / \mathrm{s}$ en la cima en $X / D=0$.

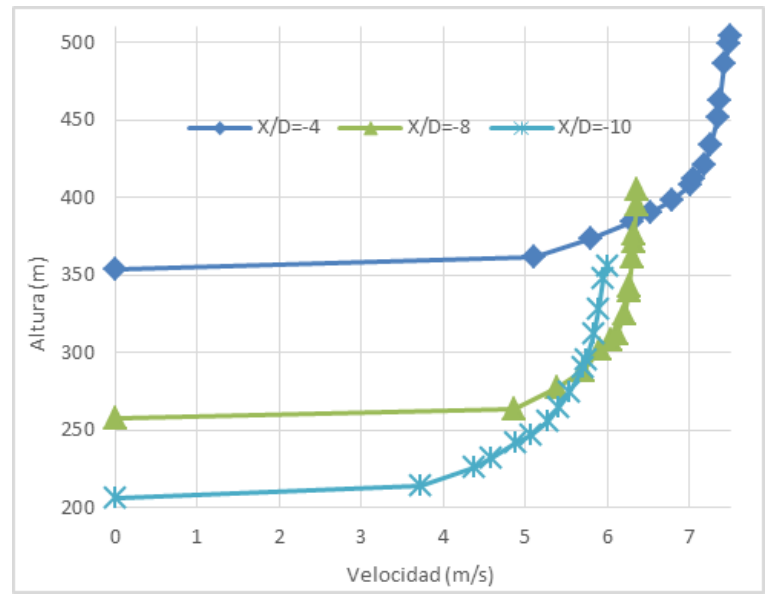

Figura 24. Perfil de velocidad en la cima de la colina $X / D=0$ en sitio de interés.

Para los perfiles de velocidad pendiente abajo; no se tiene un patrón definido de comportamiento. La velocidad decrece a medida que aumenta la distancia con respecto al centro de la colina; se presentan cambios de dirección en la velocidad, dando lugar a la recirculación del flujo en el valle. La velocidad en la zona antes mencionada es de $5.8 \mathrm{~m} / \mathrm{s}$ aproximadamente para $X / D=15$; como se puede observar en la Figura 25.

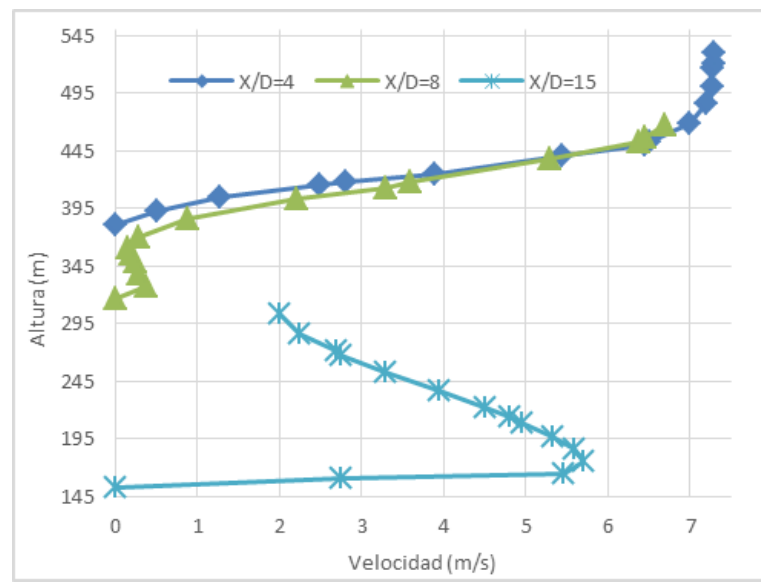

Figura 25. Perfiles de velocidad pendiente abajo en sitio de interés para $X / D=4, X / D=8$ y $X / D=15$.

Realizado un análisis similar al efectuado con la velocidad media; en la Figura 26 se muestran los contornos de energía cinética turbulenta a lo largo del dominio, con valores entre $0.003 \mathrm{~J} / \mathrm{kg}$ a $0.597 \mathrm{~J} / \mathrm{kg}$.

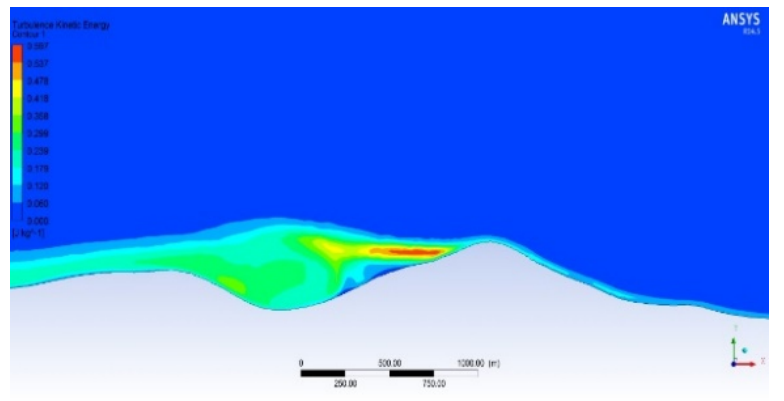

Figura 26. Contornos de energía cinética turbulenta en el dominio del sitio de interés.

Se observa un aumento de la energía cinética turbulenta a medida que disminuye la altura hasta llegar a un valor máximo cerca del suelo, para luego disminuir. En la Figura 27 se muestra el perfil de energía cinética turbulenta en la cresta de la colina $X / D=0$, con valores máximos de $0.17 \mathrm{~m}^{2} / \mathrm{s}^{2}$.

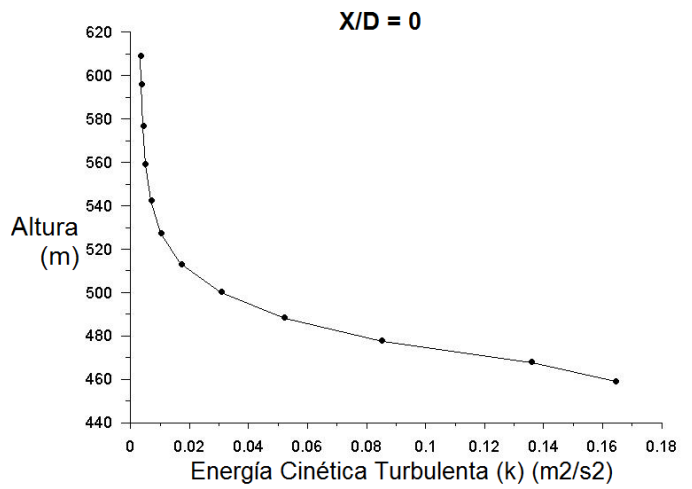

Figura 27. Energía cinética turbulenta en la cima de la colina $X / D=0$ para sitio de interés.

En cuanto a los perfiles de energía cinética turbulenta generados pendiente arriba, se puede observar perfiles homogéneos en las diferentes posiciones en la Figura 28. Los valores máximos registrados están en la posición $X / D=-4$ con un valor de $0.14 \mathrm{~m}^{2} / \mathrm{s}^{2}$.

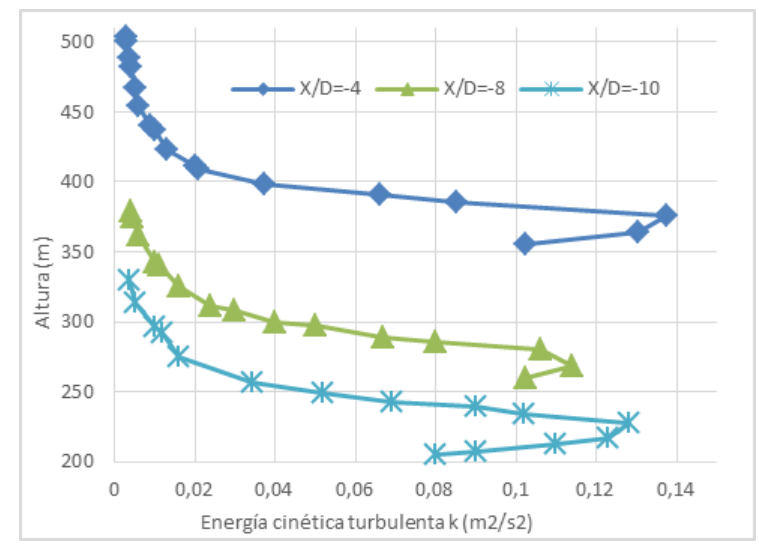

Figura 28. Perfiles de energía cinética turbulenta aguas arriba en sitio de interés para $X / D=-4, X / D=-8$ y $X / D=-10$. 
El comportamiento de la intensidad de turbulencia pendiente arriba de la loma, de acuerdo con lo expuesto en el párrafo anterior; solo se incrementan en una capa delgada cerca del suelo, por debajo de $H \approx 120 \mathrm{~m}$. En esta zona existe una mayor producción de turbulencia media debido al mayor cizallamiento del flujo; por el contrario, a mayores alturas del suelo se da una disminución de la intensidad de la turbulencia permaneciendo prácticamente constante; afirmación que concuerda en su totalidad con el estudio de Rokenes y Krogstad.

En los perfiles de energía cinética turbulenta pendiente abajo (Figura 29); no se observa un comportamiento homogéneo, los valores son considerablemente más altos que para el caso de la energía cinética turbulenta pendiente arriba; dando lugar a la separación del flujo en el valle. El incremento de la turbulencia se presenta a alturas de $H=140 \mathrm{~m}$ del suelo, para las diferentes posiciones. La energía cinética turbulenta de la posición $X / D=15$ tiene un valor de $0.26 \mathrm{~m}^{2} / \mathrm{s}^{2}$; aproximadamente dos veces mayor a la energía cinética turbulenta de la cresta y del resto del perfil de pendiente arriba de la colina en estudio.

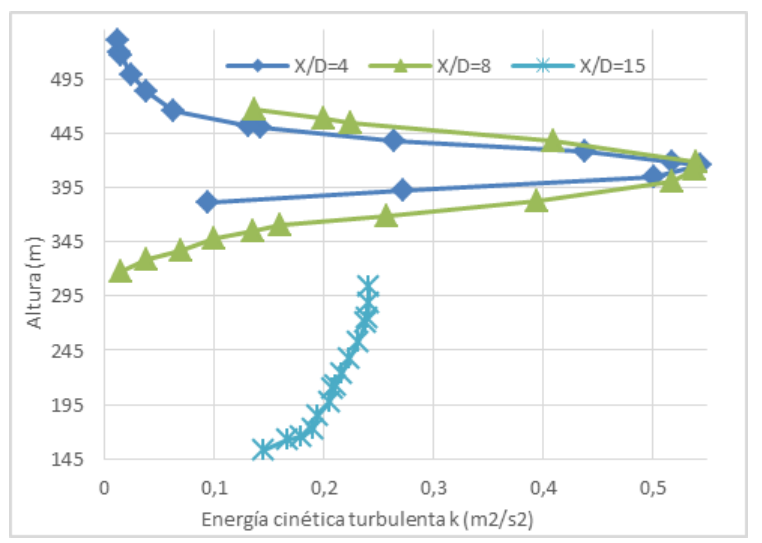

Figura 29. Perfiles de energía cinética turbulenta pendiente abajo en sitio de interés para $X / D=4, X / D=8 \mathrm{y}$ $X / D=15$.

\section{Conclusiones}

En el presente trabajo se ha realizado el estudio del flujo de aire sobre una colina que puede ser considerada como un terreno complejo; mediante simulaciones numéricas bidimensionales utilizando el paquete computacional Ansys Fluent; logrando describir el comportamiento del campo de velocidades y turbulencia correspondientes al sitio seleccionado para el estudio. Las principales conclusiones a partir de estos criterios se pueden resumir de la siguiente manera:

Se ha realizado simulaciones bidimensionales del flujo de aire sobre diversos elementos topográficos característicos de terrenos complejos, como son colinas y valles. Los resultados numéricos obtenidos en las simulaciones ofrecen una buena coincidencia, en el comportamiento de los campos de velocidades y de energía cinética turbulenta a lo largo del dominio establecido.

El procedimiento de mallado aplicado al dominio computacional de las colinas analizadas, garantizan en buena medida la exactitud de los resultados obtenidos para los campos de velocidad y energía cinética turbulenta. El dominio computacional fue estratificado en dos sectores con diferentes refinamientos, se utilizó una malla no estructurada de volúmenes finitos en forma de tetraedros. Por otro lado, las simulaciones bidimensionales aplicadas, permitieron construir una malla más fina a costos computacionales convenientes.

El modelo de turbulencia $k-\epsilon$ utilizado en las distintas simulaciones para caracterizar la turbulencia de las colinas o sitios estudiados que de acuerdocon los resultados alcanzados, resulta ser un modelo consistente para el estudio de la turbulencia en terrenos complejos.

La validación del procedimiento utilizado para el estudio del campo de viento sobre el sitio seleccionado se llevó a cabo mediante simulaciones bidimensionales en emplazamientos con datos conocidos. Los resultados numéricos obtenidos ofrecen resultados con coincidencias bastante cercanas, tanto para el campo de velocidades como para la energía cinética turbulenta; razón por la cual, se puede afirmar que el procedimiento establecido es conveniente para el estudio del campo de viento en colinas.

Se ha realizado las simulaciones del cerro «Villonaco» ubicado al sur de Ecuador; en donde se encuentra operando un parque eólico que lleva el mismo nombre y que, por sus características la colina en mención, puede ser considerada como un terreno complejo. Las simulaciones realizadas, han permitido caracterizar con cierto grado de detalle el flujo de aire en la zona.

\section{Referencias}

[1] A. E. Kasmi and C. Masson, "An extended $k-\epsilon$ model for turbulent flow through horizontal-axis wind turbines," Journal of Wind Engineering and Industrial Aerodynamics, vol. 96, no. 1, pp. $103-$ $122,2008$.

[2] R. Abajo Gálvez, "Análisis del desarrollo de la estela de un aerogenerador en terrenos complejos," Trabajo de fin de máster, ETS - Ingenieros Industriales UNED, España, 2011.

[3] S. Finardi, G. Tinarelli, P. Faggian, and G. Brusasca, "Evaluation of different wind field modeling techniques for wind energy applications over complex topography," Journal of Wind Engineering and Industrial Aerodynamics, vol. 74-76, pp. $283-294,1998$. 
[4] O. Gauravy and V. Sharad, "A review of computer models for wind flow over mountainous terrain," in International Conference on Environmental Fluid Mechanics (ICEFM), India, 2005, pp. 151-157.

[5] C. G. Justus, Vientos, rendimiento del sistema y del viento. The Franklin Institute Press, 1978.

[6] J. Kaimal and J. Finnigan, Atmospheric Boundary Layer Flows: Their Structure and Measurement. Oxford University Press, 1994.

[7] G. Teneler, "Wind flow analysis on a complex terrain: a reliability study of a CFD tool on forested area including effects of forest module," Master of Science Thesis, Gotland University, 2011.

[8] A. Griffiths and J. Middleton, "Simulations of separated flow over two-dimensional hills," Journal of wind engineering and industrial aerodynamics, vol. 98, no. 3, pp. 155-160, 2010.

[9] A. Maurizi, "Numerical simulation of turbulent flows over 2-D valleys using three versions of the $k$ - $\epsilon$ closure model," Journal of Wind Engineering and Industrial Aerodynamics, vol. 85, no. 1, pp. $59-73,2000$.

[10] J. D. Anderson and J. Wendt, Computational fluid dynamics. Springer, 1995, vol. 206.

[11] J. D. Walshe, "CFD modelling of wind flow over complex and rough terrain," Ph.D. dissertation, Loughborough University, 2003.

[12] J. Hunt, F. Tampieri, W. Weng, and D. Carruthers, "Air flow and turbulence over complex terrain: a colloquium and a computational workshop," Journal of Fluid Mechanics, vol. 227, pp. 667-688, 1991.

[13] J. A. Capote, D. Alvear, O. V. Abreu, M. Lázaro, and P. Espina, "Influencia del modelo de turbulencia y del refinamiento de la discretización espacial en la exactitud de las simulaciones computacionales," Revista Internacional de Métodos Numéricos para Cálculo y Diseño en Ingeniería, vol. 24 , no. 3 , pp. 227-245, 2008.

[14] W. Gómez and L. Llano, "Simulación del viento atmosférico y aplicación experimental," Ciencia e Ingeniería Neogranadina, vol. 16, no. 1, pp. 5-14, 2006.

[15] J. M. Prospathopoulos, E. S. Politis, K. G. Rados, and P. K. Chaviaropoulos, "Evaluation of the effects of turbulence model enhancements on wind turbine wake predictions," Wind Energy, vol. 14, no. 2, pp. 285-300, 2011.

[16] J. O'Sullivan, R. Pecnik, and G. Iaccarino, "Investigating turbulence in wind flow over complex terrain," in Proceedings of the Summer Program, Stanford University, 2010, pp. 129-139.

[17] J. Forthofer and B. Butler, "Differences in simulated fire spread over askervein hill using two advanced wind models and a traditional uniform wind field," in The Fire enviroment - Innovations, Management, and Policy, B. Butler and W. Cook, Eds., Rocky Mountain Research. Destin, FL: United States Department of Agriculture, 26-30 March 2007, pp. 123-127.

[18] H. G. Ollague-Armijos and H. E. Crespo-Azanza, "Estudio y evaluación de los parámetros de operación del proyecto eólico Villonaco de la provincia de Loja - Ecuador," Trabajo de conclusión de curso, Universidad Politécnica Salesiana, Cuenca - Ecuador, 2014.

[19] CELEC. Redición de cuentas 2014. CELEC. Cuenca, Ecuador. [Online]. Available: https: //www.celec.gob.ec/

[20] K. Rokenes, "Investigation of terrain effects with respect to wind farm siting," Ph.D. dissertation, Norwegian University of Science and Technology, Norway, 2009. 